\title{
An Algorithm for Biometric Authentication Based on the Model of Non-Stationary Random Processes
}

\author{
Vladimir B. Balakirsky, Anahit R. Ghazaryan, and A.J. Han Vinck \\ Institute for Experimental Mathematics, Ellernstr. 29, 45326 Essen, Germany \\ v_b_balakirsky@rambler.ru, a_ghazaryan@rambler.ru, vinck@iem.uni-due.de
}

\begin{abstract}
We present a biometric authentication algorithm with 3 possible outputs: accept the claimed identity, reject the claimed identity, and no answer. The algorithm is based on the model of non-stationary random processes and transformations of realizations of the processes to $q$-ary vectors whose components are uniformly distributed over the set $\{0, \ldots, q-1\}$, where $q$ is fixed. An application of the algorithm brings the false acceptance and the false rejection rates that do not depend on the probability distribution over the templates space and exponentially decrease with the number of independent biometric parameters available to an observer.
\end{abstract}

\section{Introduction}

There are many situations where an object is characterized by some list of parameters of different nature. Examples include biometric measurements when the list can consist of the height of the person, the color of hairs, the weight, etc. 1. These parameters are written in a vector and stored in a database under the name of the person. The claimed identity of a person is then checked by comparison of the vector consisting of results of his measurements and the corresponding vector fetched from the database. The difficulties of organizing such a procedure are caused by the fact that independent measurements of the same parameter of a person usually bring different results. Therefore one has to develop criteria that specify contributions of non-equal components of two vectors to the binary decision: the claimed identity should be either accepted or rejected.

By the point that components of two vectors under considerations are measured in different units, one has to find an artificial space where results of two measurements can be compared. We develop mathematical model of nonstationary random processes assuming that components are outcomes of random experiments with specified probability density functions (PDF's). Different components are assumed to be independent. The PDF also determines the probability distribution function (PD) whose value belongs to the interval $[0,1]$ for any PDF. This observation allows us to unify parameters: the value of each parameter is considered as the argument of the corresponding PD and it is replaced by the value of function. 
Our analysis of the approach above is presented via evaluation of the false acceptance and the false rejection rates, where the false acceptance rate is the probability that the claimed identity is accepted while people are different, and the false rejection rate is the probability that the claimed identity is not accepted while parameters are measured for the same person. The basic result is the point that our approach guarantees these rates to be exponentially decreasing functions with the number of parameters under certain conditions. Furthermore, we prove a universal estimate on the false rejection rate that does not depend on the PDF's of parameters.

\section{Statement of the Problem and a General Authentication Algorithm}

We will use notation of probability theory where capital letters denote random variables and random processes.

Let $\mathbf{X}=\left(X^{(1)}, \ldots, X^{(n)}\right)$ and $\mathbf{Y}=\left(Y^{(1)}, \ldots, Y^{(n)}\right)$ be random processes, where $\left(X^{(1)}, Y^{(1)}\right), \ldots,\left(X^{(n)}, Y^{(n)}\right)$ are independent pairs of random variables. Suppose that the PDF's of random variables $X^{(t)}$ and $Y^{(t)}$ be given as functions $f_{\mathrm{x}}^{(t)}$ and $f_{\mathrm{y}}^{(t)}$ in such a way that the PD's

$$
F_{\mathrm{x}}^{(t)}(x) \triangleq \int_{-\infty}^{x} f_{\mathrm{x}}^{(t)}\left(x^{\prime}\right) d x^{\prime}, \quad F_{\mathrm{x}}^{(t)}(y) \triangleq \int_{-\infty}^{y} f_{\mathrm{y}}^{(t)}\left(y^{\prime}\right) d y^{\prime}
$$

are strictly increasing functions over the set R. This property will be indicated as $F_{\mathrm{x}}^{(t)}, F_{\mathrm{y}}^{(t)} \in \mathcal{F}$, where $F_{\mathrm{x}}^{(t)}=\left(F_{\mathrm{x}}^{(t)}(x), x \in \mathrm{R}\right)$ and $F_{\mathrm{y}}^{(t)}=\left(F_{\mathrm{y}}^{(t)}(y), y \in \mathrm{R}\right)$.

Let $f_{\mathrm{y} \mid \mathrm{x}}^{(t)}(y \mid x), x, y \in \mathrm{R}$, be introduced as a kernel of the integral transformation

$$
\int_{\mathrm{R}} f_{\mathrm{x}}^{(t)}(x) f_{\mathrm{y} \mid \mathrm{x}}^{(t)}(y \mid x) d x=f_{\mathrm{y}}^{(t)}(y), \text { for all } y \in \mathrm{R},
$$

and let

$$
F_{\mathrm{y} \mid \mathrm{x}}^{(t)}(y \mid x) \triangleq \int_{-\infty}^{y} f_{\mathrm{y} \mid \mathrm{x}}^{(t)}\left(y^{\prime} \mid x\right) d y^{\prime}, \quad y \in \mathrm{R},
$$

denote the conditional PD for all $x \in \mathrm{R}$.

Suppose that $\mathbf{x}=\left(x^{(1)}, \ldots, x^{(n)}\right) \in \mathrm{R}^{n}$ and $\mathbf{y}=\left(y^{(1)}, \ldots, y^{(n)}\right) \in \mathrm{R}^{n}$ are realizations of the processes $\mathbf{X}$ and $\mathbf{Y}$. Denote

$$
\begin{aligned}
& \left(f_{\mathrm{x}}(\mathbf{x}), f_{\mathrm{y}}(\mathbf{y}), f_{\mathrm{y} \mid \mathrm{x}}(\mathbf{y} \mid \mathbf{x})\right) \\
\triangleq & \left(\prod_{t=1}^{n} f_{\mathrm{x}}^{(t)}\left(x^{(t)}\right), \prod_{t=1}^{n} f_{\mathrm{y}}^{(t)}\left(y^{(t)}\right), \prod_{t=1}^{n} f_{\mathrm{y} \mid \mathrm{x}}^{(t)}\left(y^{(t)} \mid x^{(t)}\right)\right),
\end{aligned}
$$

and formulate two problems.

I: Is it true that $\mathbf{x}$ and $\mathbf{y}$ are realizations of independent processes and the value of the joint PDF is expressed as $f_{\mathrm{x}}(\mathbf{x}) f_{\mathrm{y}}(\mathbf{y})$ ? The question corresponds to the case when the vector $\mathbf{x}$ contains biometrics of a person from the database, and the vector $\mathbf{y}$ contains biometrics of a person chosen at random. 
D: Is it true that $\mathbf{x}$ and $\mathbf{y}$ are realizations of dependent processes and the value of the joint PDF is expressed as $f_{\mathrm{x}}(\mathbf{x}) f_{\mathrm{y} \mid \mathrm{x}}(\mathbf{y} \mid \mathbf{x})$ ? The question corresponds to the case when the vector $\mathbf{y}$ contains a noisy version of biometrics $\mathbf{x}$ of some person from the database.

General solutions to the $\mathbf{I} / \mathbf{D}$ problems can be specified by sets $\mathcal{I}, \mathcal{D} \subset \mathrm{R}^{n} \times$ $\mathrm{R}^{n}$. The answers are positive if $(\mathbf{x}, \mathbf{y}) \in \mathcal{I}$ and $(\mathbf{x}, \mathbf{y}) \in \mathcal{D}$ in the $\mathbf{I}$ and the D problem, respectively. The peculiarities of our situation as compared to the situation considered in the hypotheses testing direction [2] are caused by the fact that sets $\mathcal{I}$ and $\mathcal{D}$ have to be disjoint. In other words, these sets cannot be assigned independently, and an algorithm for testing the independence in the $\mathbf{I}$ problem depends on the specification of the dependency in the $\mathbf{D}$ problem. However, one can overcome these difficulties if the "no answer" output is allowed in the case when $(\mathbf{x}, \mathbf{y}) \in \mathcal{I} \cap \mathcal{D}$. We accept such an assumption and translate it to a general authentication algorithm as follows. Given the claim that $\mathbf{x}$ and $\mathbf{y}$ describe biometrics of the same person, assign two sets, $\mathcal{I}, \mathcal{D} \subset \mathrm{R}^{n} \times \mathrm{R}^{n}$, and denote

$$
\overline{\mathcal{I}} \triangleq\left(\mathrm{R}^{n} \times \mathrm{R}^{n}\right) \backslash \mathcal{I}, \quad \overline{\mathcal{D}} \triangleq\left(\mathrm{R}^{n} \times \mathrm{R}^{n}\right) \backslash \mathcal{D} .
$$

Set

$$
\text { Decision }= \begin{cases}\text { "accept the claim", } & \text { if }(\mathbf{x}, \mathbf{y}) \in \overline{\mathcal{I}} \cap \mathcal{D}, \\ \text { "no answer", } & \text { if }(\mathbf{x}, \mathbf{y}) \in \mathcal{I} \cap \mathcal{D}, \\ \text { "reject the claim", } & \text { if }(\mathbf{x}, \mathbf{y}) \in \overline{\mathcal{D}}\end{cases}
$$

Notice that the acceptance and the rejection decisions in (4) are non-symmetric, since we reject the identity if $(\mathbf{x}, \mathbf{y}) \in\{\mathcal{I} \cap \overline{\mathcal{D}}, \overline{\mathcal{I}} \cap \overline{\mathcal{D}}\}$.

If only the $\mathbf{I}$ problem is considered, then it can be viewed as testing of hypotheses $f=f_{\mathrm{x}, \mathrm{y}}$ or $f \neq f_{\mathrm{x}, \mathrm{y}}$, and the performance of an $\mathbf{I}$ algorithm is characterized by the error probability

$$
\Pi_{\mathrm{i}}(\mathcal{I}) \triangleq \int_{\mathrm{R}^{n} \times \mathrm{R}^{n}} f_{\mathrm{x}}(\mathbf{x}) f_{\mathrm{y}}(\mathbf{y}) \chi\{(\mathbf{x}, \mathbf{y}) \notin \mathcal{I}\} d \mathbf{x} d \mathbf{y},
$$

where $\chi$ denotes the indicator function: $\chi\{S\}=1$ if the statement $S$ is true and $\chi\{S\}=0$ otherwise. Similarly, if only the $\mathbf{D}$ problem is considered, then it can be viewed as testing of hypotheses $f=f_{\mathrm{x}, \mathrm{y} \mid \mathrm{x}}$ or $f \neq f_{\mathrm{x}, \mathrm{y} \mid \mathrm{x}}$, and the performance of a $\mathbf{D}$ algorithm is characterized by the error probability

$$
\Pi_{\mathrm{d}}(\mathcal{D}) \triangleq \int_{\mathrm{R}^{n} \times \mathrm{R}^{n}} f_{\mathrm{x}}(\mathbf{x}) f_{\mathrm{y} \mid \mathrm{x}}(\mathbf{y} \mid \mathbf{x}) \chi\{(\mathbf{x}, \mathbf{y}) \notin \mathcal{D}\} d \mathbf{x} d \mathbf{y} .
$$

Certainly, assignments $\mathcal{I}, \mathcal{D}=\mathrm{R}^{n} \times \mathrm{R}^{n}$ bring the probabilities $\Pi_{\mathrm{i}}(\mathcal{I})$ and $\Pi_{\mathrm{d}}(\mathcal{D})$ equal to zero. However, combining the $\mathbf{I}$ and the $\mathbf{D}$ problems impose three hypotheses: 1) $f=f_{\mathrm{x}, \mathrm{y}}$; 2) $f=f_{\mathrm{x}, \mathrm{y} \mid \mathrm{x}}$; 3) $f \neq f_{\mathrm{x}, \mathrm{y}}$ and $f \neq f_{\mathrm{x}, \mathrm{y} \mid \mathrm{x}}$. Then the performance is also characterized by the probabilities

$$
\begin{aligned}
\Pi_{\mathrm{id}}(\mathcal{D}) & \triangleq \int_{\mathrm{R}^{n} \times \mathrm{R}^{n}} f_{\mathrm{x}}(\mathbf{x}) f_{\mathrm{y}}(\mathbf{y}) \chi\{(\mathbf{x}, \mathbf{y}) \in \mathcal{D}\} d \mathbf{x} d \mathbf{y} \\
\Pi_{\mathrm{di}}(\mathcal{I}) & \triangleq \int_{\mathrm{R}^{n} \times \mathrm{R}^{n}} f_{\mathbf{x}}(\mathbf{x}) f_{\mathrm{y} \mid \mathbf{x}}(\mathbf{y} \mid \mathbf{x}) \chi\{(\mathbf{x}, \mathbf{y}) \in \mathcal{I}\} d \mathbf{x} d \mathbf{y} .
\end{aligned}
$$


Roughly speaking, we want all four probabilities to be small. For example, the problem can be formulated as follows: given the PDF's satisfying (1), (2), construct sets $\mathcal{I}, \mathcal{D}$ in such a way that

$$
\max \left\{\Pi_{\mathrm{i}}(\mathcal{I}), \Pi_{\mathrm{d}}(\mathcal{D}), \Pi_{\mathrm{id}}(\mathcal{D}), \Pi_{\mathrm{di}}(\mathcal{I})\right\} \rightarrow \min .
$$

We will present a specific construction of the sets $\mathcal{I}, \mathcal{D}$ that are parameterized by non-negative numbers $\delta_{0}$ and $\delta_{1}$. Independently of the PDF's, the probabilities $\Pi_{\mathrm{i}}(\mathcal{I})$ and $\Pi_{\mathrm{d}}(\mathcal{D})$ are expressed through $\delta_{0}, \delta_{1}$, and auxiliary integer $q$, and they exponentially decrease with the number of observations $n$. Notice also that the same value of $\Pi_{\mathrm{i}}(\mathcal{I})$ and $\Pi_{\mathrm{d}}(\mathcal{D})$ can be obtained for different parameters $\delta_{0}$ and $\delta_{1}$. These parameters have to be assigned to minimize $\Pi_{\mathrm{id}}(\mathcal{D})$ and $\Pi_{\mathrm{di}}(\mathcal{I})$. The result of optimization depends on the PDF's under considerations.

\section{The $\xi$ Transformations of Random Variables and the Conditional Distortions Between Biometric Vectors}

Suppose that $X$ is a continuous random variables with the $\mathrm{PD} F \in \mathcal{F}$ and let $q$ be a fixed positive integer. Denote $\mathcal{Q} \triangleq\{0, \ldots, q-1\}$ and

$$
\mathcal{Q}^{(n)} \triangleq \bigcup_{c=0}^{n(q-1)}\{c /(n q)\}
$$

Introduce the $\xi_{\mathrm{x}}, \xi_{\mathrm{y}}$, and $\xi_{\mathrm{y} \mid \mathrm{x}}$ transformations in the following way. For all $x, y \in \mathrm{R}$, let

$$
\xi_{\mathrm{x}}(x)=\left\lfloor q F_{\mathrm{x}}(x)\right\rfloor, \quad \xi_{\mathrm{y}}(y)=\left\lfloor q F_{\mathrm{y}}(y)\right\rfloor, \quad \xi_{\mathrm{y} \mid \mathrm{x}}(y \mid x)=\left\lfloor q F_{\mathrm{y} \mid \mathrm{x}}(y \mid x)\right\rfloor,
$$

where $\lfloor z\rfloor$ denotes the integer part of $z \in \mathrm{R}$.

The $\xi_{\mathrm{x}}$ transformation is illustrated in Fig. 11. Notice that equalities (10) determine a uniform distribution over the set $\mathcal{Q}$ :

$$
\operatorname{Pr}\left\{\xi_{\mathrm{x}}(X)=i\right\}=\int_{F_{\mathrm{X}}^{-1}(i / q)}^{F_{\mathrm{X}}^{-1}((i+1) / q)} f_{\mathrm{X}}(x) d x=1 / q, \quad i \in \mathcal{Q} .
$$

Furthermore, for all $x \in \mathrm{R}$,

$$
\operatorname{Pr}\left\{\xi_{\mathrm{y} \mid \mathrm{x}}(Y \mid x)=j\right\}=\int_{F_{\mathrm{y} \mid \mathrm{x}}^{-1}(j / q \mid x)}^{F_{\mathrm{y} \mid \mathrm{x}}^{-1}((j+1) / q \mid x)} f_{\mathrm{y} \mid \mathrm{x}}(y \mid x) d y=1 / q, \quad j \in \mathcal{Q} .
$$

Let us apply transformations (10) to components of the processes $\mathbf{X}$ and $\mathbf{Y}$ by substituting $\left(F_{\mathrm{x}}, F_{\mathrm{y}}, F_{\mathrm{y} \mid \mathrm{x}}(x)\right)=\left(F_{\mathrm{x}}^{(t)}, F_{\mathrm{y}}^{(t)}, F_{\mathrm{y} \mid \mathrm{x}}^{(t)}\left(x^{(t)}\right)\right), t=1, \ldots, n$. Set

$$
\begin{aligned}
& \Delta_{\mathrm{i}}\left(x^{(t)}, y^{(t)}\right) \triangleq \frac{\left|\xi_{\mathrm{x}}\left(x^{(t)}\right)-\xi_{\mathrm{y}}\left(y^{(t)}\right)\right|}{q}, \\
& \Delta_{\mathrm{d}}\left(x^{(t)}, y^{(t)}\right) \triangleq \frac{\left|\xi_{\mathrm{x}}\left(x^{(t)}\right)-\xi_{\mathrm{y} \mid \mathrm{x}}\left(y^{(t)} \mid x^{(t)}\right)\right|}{q},
\end{aligned}
$$


and define

$$
\Delta_{\mathrm{i}}(\mathbf{x}, \mathbf{y}) \triangleq \sum_{t=1}^{n} \Delta_{\mathrm{i}}\left(x^{(t)}, y^{(t)}\right), \quad \Delta_{\mathrm{d}}(\mathbf{x}, \mathbf{y}) \triangleq \sum_{t=1}^{n} \Delta_{\mathrm{d}}\left(x^{(t)}, y^{(t)}\right)
$$

as the conditional distortions between $\mathbf{x}$ and $\mathbf{y}$.

Introduce the $(\Delta \mid \xi)$-authentication algorithm as follows. Fix two thresholds, $\delta_{0}$ and $\delta_{1}$. Given the claim that $\mathbf{x}$ and $\mathbf{y}$ describe biometrics of the same person, compute $\Delta_{\mathrm{i}}(\mathbf{x}, \mathbf{y}), \Delta_{\mathrm{d}}(\mathbf{x}, \mathbf{y})$, and denote

$$
\begin{aligned}
& \mathcal{I} \triangleq\left\{(\mathbf{x}, \mathbf{y}) \in \mathrm{R}^{n} \times \mathrm{R}^{n}: \Delta_{\mathrm{i}}(\mathbf{x}, \mathbf{y}) \in\left(n \delta_{0}, n \delta_{1}\right)\right\}, \\
& \mathcal{D} \triangleq\left\{(\mathbf{x}, \mathbf{y}) \in \mathrm{R}^{n} \times \mathrm{R}^{n}: \Delta_{\mathrm{d}}(\mathbf{x}, \mathbf{y}) \in\left(n \delta_{0}, n \delta_{1}\right)\right\} .
\end{aligned}
$$

Follow rules (4), where sets $\overline{\mathcal{I}}, \overline{\mathcal{D}}$ are defined in (3).

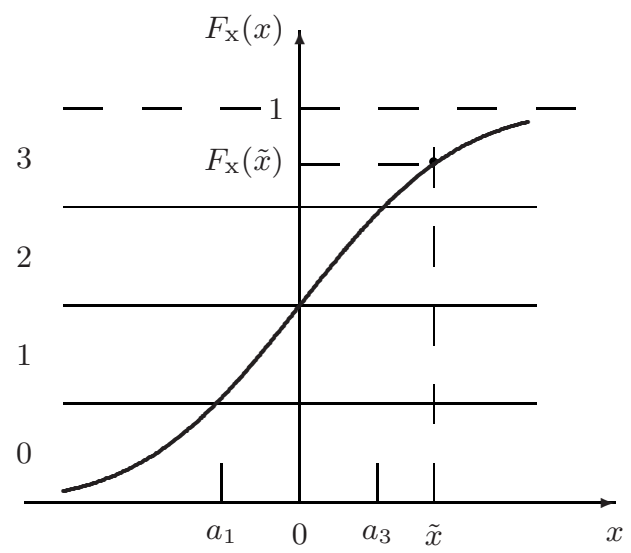

Fig. 1. Example of the transformation $\tilde{x} \rightarrow \xi_{\mathrm{x}}(\tilde{x})=3$ for $q=4$, where $a_{1}=F_{\mathrm{x}}^{-1}(1 / 4)$ and $a_{3}=F_{\mathrm{x}}^{-1}(3 / 4)$

\section{Performance of the $(\Delta \mid \xi)$-algorithm}

Given a PD over the set $\mathcal{Q}^{(1)}$ specified by some vector $\mathbf{p}=\left(p_{0}, \ldots, p_{q-1}\right)$, introduce the generating function

$$
G_{\mathbf{p}}(w) \triangleq \sum_{c=0}^{q-1} p_{c} w^{c}
$$

where $w$ is a formal variable, and express the $n$-th power of the polynomial $G_{\mathbf{p}}(w)$ as

$$
\left(G_{\mathbf{p}}(w)\right)^{n}=\sum_{\delta \in \mathcal{Q}^{(n)}} \Psi_{\mathbf{p}}(\delta) w^{n q \delta}
$$


The equalities (11), (12) immediately bring the following universal statement (the claimed performance does not depend on the PDF's under considerations).

Proposition 1. Let $\boldsymbol{\beta}=\left(\beta_{0}, \ldots, \beta_{q-1}\right)$ be the vector having components

$$
\beta_{c} \triangleq \frac{1}{q^{2}}|\{i, j \in \mathcal{Q}:|i-j|=c\}|=\frac{q-c}{q^{2}} \cdot\left\{\begin{array}{l}
1, \text { if } c=0, \\
2, \text { if } c \in\{1, \ldots, q-1\} .
\end{array}\right.
$$

If the sets $\mathcal{I}$ and $\mathcal{D}$ are defined by (15), (16), then, for all $\left(F_{\mathrm{x}}^{(1)}, \ldots, F_{\mathrm{x}}^{(n)}\right)$, $\left(F_{\mathrm{y}}^{(1)}, \ldots, F_{\mathrm{y}}^{(n)}\right),\left(F_{\mathrm{y} \mid \mathrm{x}}^{(1)}\left(x^{(1)}\right), \ldots, F_{\mathrm{y} \mid \mathrm{x}}^{(n)}\left(x^{(n)}\right)\right) \in \mathcal{F}^{n}$,

$$
\Pi_{\mathrm{i}}(\mathcal{I})=\Pi_{\mathrm{d}}(\mathcal{D})=\sum_{\delta \in \mathcal{Q}^{(n)} \cap\left(\delta_{0}, \delta_{1}\right)} \Psi_{\boldsymbol{\beta}}(\delta),
$$

where the probabilities $\Pi_{\mathrm{i}}(\mathcal{I})$ and $\Pi_{\mathrm{d}}(\mathcal{D})$ are defined in (5), (6) .

Results of the analysis of the probabilities $\Pi_{\mathrm{id}}(\mathcal{D})$ and $\Pi_{\mathrm{di}}(\mathcal{I})$ are less universal in a sense that they depend on the specific PDF's. For all $t=1, \ldots, n$, introduce two PD's over the set $\mathcal{Q}^{(1)}$ by the vectors $\boldsymbol{\beta}_{\mathrm{id}}^{(t)}=\left(\beta_{\mathrm{id}, 0}^{(t)}, \ldots, \beta_{\mathrm{id}, q-1}^{(t)}\right)$ and $\boldsymbol{\beta}_{\mathrm{di}}^{(t)}=$ $\left(\beta_{\mathrm{di}, 0}^{(t)}, \ldots, \beta_{\mathrm{di}, q-1}^{(t)}\right)$, where

$$
\begin{aligned}
& \beta_{\mathrm{id}, c}^{(t)}=\int_{\mathrm{R} \times \mathrm{R}} f_{\mathrm{x}}^{(t)}(x) f_{\mathrm{y}}^{(t)}(y) \chi\left\{\left|\xi_{\mathrm{x}}(x)-\xi_{\mathrm{y} \mid \mathrm{x}}(y \mid x)\right|=c\right\} d x d y, \\
& \beta_{\mathrm{di}, c}^{(t)}=\int_{\mathrm{R} \times \mathrm{R}} f_{\mathrm{x}}^{(t)}(x) f_{\mathrm{y} \mid \mathrm{x}}^{(t)}(y \mid x) \chi\left\{\left|\xi_{\mathrm{x}}(x)-\xi_{\mathrm{y}}(y)\right|=c\right\} d x d y .
\end{aligned}
$$

For $\mathbf{p}^{(t)}=\boldsymbol{\beta}_{\mathrm{id}}^{(t)}, \boldsymbol{\beta}_{\mathrm{di}}^{(t)}, t=1, \ldots, n$, let us express the product of $n$ polynomials $G_{\mathbf{p}^{(1)}}(w), \ldots, G_{\mathbf{p}^{(n)}}(w)$ as

$$
\prod_{t=1}^{n} G_{\mathbf{p}^{(t)}}(w)=\sum_{\delta \in \mathcal{Q}^{(n)}} \Psi_{\mathbf{p}^{(1)}, \ldots, \mathbf{p}^{(n)}}(\delta) w^{n q \delta} .
$$

Proposition 2. If the sets $\mathcal{I}$ and $\mathcal{D}$ are defined by (15), (16), then, for all $\left(F_{\mathrm{x}}^{(1)}, \ldots, F_{\mathrm{x}}^{(n)}\right),\left(F_{\mathrm{y}}^{(1)}, \ldots, F_{\mathrm{y}}^{(n)}\right),\left(F_{\mathrm{y} \mid \mathrm{x}}^{(1)}\left(x^{(1)}\right), \ldots, F_{\mathrm{y} \mid \mathrm{x}}^{(n)}\left(x^{(n)}\right)\right) \in \mathcal{F}^{n}$,

$$
\begin{aligned}
\Pi_{\mathrm{id}}(\mathcal{D}) & =\sum_{\delta \in \mathcal{Q}^{(n)} \cap\left(\delta_{0}, \delta_{1}\right)} \Psi_{\boldsymbol{\beta}_{\mathrm{id}}^{(1)}, \ldots, \boldsymbol{\beta}_{\mathrm{id}}^{(n)}}(\delta), \\
\Pi_{\mathrm{di}}(\mathcal{I}) & =\sum_{\delta \in \mathcal{Q}^{(n)} \cap\left(\delta_{0}, \delta_{1}\right)} \Psi_{\boldsymbol{\beta}_{\mathrm{di}}^{(1)}, \ldots, \boldsymbol{\beta}_{\mathrm{di}}^{(n)}}(\delta),
\end{aligned}
$$

where the probabilities $\Pi_{\mathrm{id}}(\mathcal{D})$ and $\Pi_{\mathrm{di}}(\mathcal{I})$ are defined in (7), (8). In particular, if $\boldsymbol{\beta}_{\mathrm{id}}^{(t)}=\boldsymbol{\beta}_{\mathrm{id}}$ and $\boldsymbol{\beta}_{\mathrm{di}}^{(t)}=\boldsymbol{\beta}_{\mathrm{di}}$ for all $t=1, \ldots, n$, then

$$
\begin{aligned}
\Pi_{\mathrm{id}}(\mathcal{D}) & =\sum_{\delta \in \mathcal{Q}^{(n)} \cap\left(\delta_{0}, \delta_{1}\right)} \Psi_{\boldsymbol{\beta}_{\mathrm{id}}}(\delta), \\
\Pi_{\mathrm{di}}(\mathcal{I}) & =\sum_{\delta \in \mathcal{Q}^{(n)} \cap\left(\delta_{0}, \delta_{1}\right)} \Psi_{\boldsymbol{\beta}_{\mathrm{di}}}(\delta) .
\end{aligned}
$$




\section{Numerical Illustrations}

Let $\left(\mathrm{g}_{m, \sigma^{2}}(x), x \in \mathrm{R}\right)$ denote a Gaussian PDF with the mean $m$ and the variance $\sigma^{2}$. We also write $\mathrm{g}_{\sigma^{2}}(x) \triangleq \mathrm{g}_{\sigma^{2}}(x \mid 0)$ and denote the corresponding Gaussian PD by $\left(\mathrm{G}_{\sigma^{2}}(x), x \in \mathrm{R}\right)$. Suppose that $q=4$ and that

$$
\left(f_{\mathrm{x}}^{(t)}(x), f_{\mathrm{y}}^{(t)}(y), f_{\mathrm{y} \mid \mathrm{x}}^{(t)}(y \mid x)\right)=\left(\mathrm{g}_{2}(x), \mathrm{g}_{2.5}(y), \mathrm{g}_{x, 0.5}(y)\right)
$$

for all $t=1, \ldots, n$.

Notice that

$$
\begin{aligned}
& \left(G_{2}^{-1}(1 / 4), G_{2}^{-1}(2 / 4), G_{2}^{-1}(3 / 4)\right)=(-0.954,0,+0.954), \\
& \left(G_{2.5}^{-1}(1 / 4), G_{2.5}^{-1}(2 / 4), G_{2.5}^{-1}(3 / 4)\right)=(-1.066,0,+1.066), \\
& \left(G_{0.5}^{-1}(1 / 4), G_{0.5}^{-1}(2 / 4), G_{0.5}^{-1}(3 / 4)\right)=(-0.477,0,+0.477) .
\end{aligned}
$$

To solve the $\mathbf{I}$ problem we map the $t$-th components of the vectors $\mathbf{x}$ and $\mathbf{y}$ to the $q$-ary elements according to the rules:

$$
\begin{aligned}
& \xi_{\mathrm{x}}\left(x^{(t)}\right)=\left\{\begin{array}{l}
0, \text { if } x^{(t)} \leq-0.954, \\
1, \text { if } x^{(t)} \in(-0.954,0], \\
2, \text { if } x^{(t)} \in(0,+0.954), \\
3, \text { if } x^{(t)} \geq+0.954,
\end{array}\right. \\
& \xi_{\mathrm{y}}\left(y^{(t)}\right)=\left\{\begin{array}{l}
0, \text { if } y^{(t)} \leq-1.066, \\
1, \text { if } y^{(t)} \in(-1.066,0], \\
2, \text { if } y^{(t)} \in(0,+1.066), \\
3, \text { if } y^{(t)} \geq+1.066 .
\end{array}\right.
\end{aligned}
$$

To solve the $\mathbf{D}$ problem we map the $t$-th component of the vector $\mathbf{x}$ to the $q$-ary element according to (21) and replace (22) with the rule:

$$
\xi_{\mathrm{y} \mid \mathrm{x}}\left(y^{(t)} \mid x^{(t)}\right)=\left\{\begin{array}{l}
0, \text { if } y^{(t)}-x^{(t)} \leq-0.477, \\
1, \text { if } y^{(t)}-x^{(t)} \in(-0.477,0], \\
2, \text { if } y^{(t)}-x^{(t)} \in(0,+0.477), \\
3, \text { if } y^{(t)}-x^{(t)} \geq+0.477 .
\end{array}\right.
$$

The constructed $q$-ary vectors are then used to determine the values of the conditional distortions and their comparisons with the thresholds.

One can check that, for any vector $\mathbf{p}$ specifying a PD on the set $\mathcal{Q}^{(1)}$, the PD $\left(\Psi_{\mathbf{p}}(\delta), \delta \in \mathcal{Q}^{(n)}\right)$ simulates the binomial distribution over the set $\mathcal{Q}^{(n)}$ with the mean

$$
\delta(\mathbf{p}) \triangleq \sum_{c=0}^{q-1}(c / q) p_{c} .
$$

These PD's determine the performance of the $\Delta \mid \xi$-algorithm according to (17) and (18), (19). An example for $(n, q)=(32,4)$ and the PDF's defined by (20) is shown in Fig 2 where we also illustrate possible assignments of parameters $\delta_{0}$ 
and $\delta_{1}$ under the conditions $\Pi_{\mathrm{id}}(\mathcal{D}) \approx \Pi_{\mathrm{i}}(\mathcal{I})$ and $\Pi_{\mathrm{di}}(\mathcal{I}) \approx \Pi_{\mathrm{d}}(\mathcal{D})$. Notice that in this case,

$$
\begin{aligned}
\boldsymbol{\beta}_{\mathrm{id}} & =(0.666,0.320,0.013,0.000), \\
\boldsymbol{\beta} & =(0.250,0.375,0.250,0.125), \\
\boldsymbol{\beta}_{\mathrm{di}} & =(0.094,0.222,0.288,0.396)
\end{aligned}
$$

and $\left(\delta\left(\boldsymbol{\beta}_{\text {id }}\right), \delta(\boldsymbol{\beta}), \delta\left(\boldsymbol{\beta}_{\mathrm{di}}\right)\right)=(0.188,0.312,0.498)$. As a result one can reliably distinguish between hypotheses under consideration.

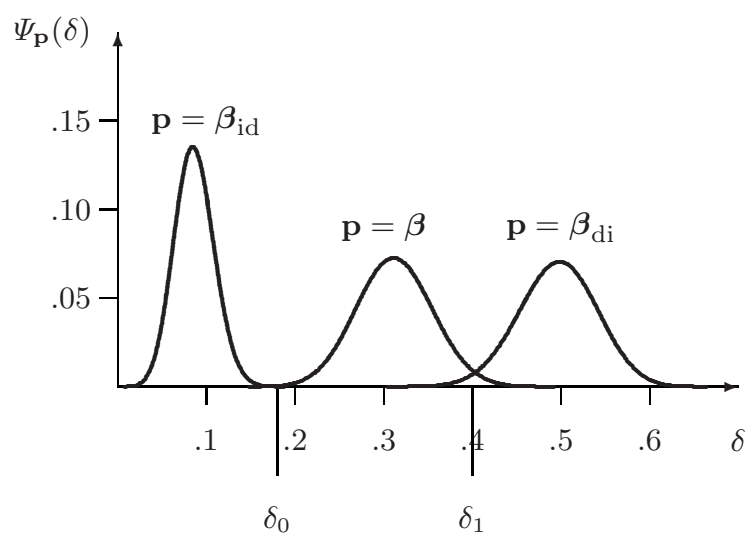

Fig. 2. The probabilities $\Psi_{\mathbf{p}}(\delta)$ for $(n, q)=(32,4)$; the PDF's are defined in (20)

\section{Conclusion}

Let us summarize the main ideas included into our analysis.

1. Suppose that the data consist of the height of a person, $\mathrm{h}$ (in centimeters), and the weight, w (in kilograms). If realizations of the noise of measurements are independent, then the conditional probability of a certain pair of parameters for a person $\mathrm{P}$ is expressed as

$$
\begin{aligned}
& \operatorname{Pr}\left\{(\hat{\mathrm{H}}, \hat{\mathrm{W}})=(\hat{\mathrm{h}}, \hat{\mathrm{w}}) \mid\left(\mathrm{H}_{\mathrm{P}}, \mathrm{W}_{\mathrm{P}}\right)=\left(\mathrm{h}_{\mathrm{P}}, \mathrm{w}_{\mathrm{P}}\right)\right\} \\
= & \operatorname{Pr}_{\mathrm{h}}\left\{\operatorname{Noise}_{\mathrm{h}}\left(\hat{\mathrm{h}}-\mathrm{h}_{\mathrm{P}}\right)\right\} \underset{\mathrm{w}}{\operatorname{Pr}}\left\{\operatorname{Noise}_{\mathrm{w}}\left(\hat{\mathrm{w}}-\mathrm{w}_{\mathrm{P}}\right)\right\} .
\end{aligned}
$$

Notice that the product above combines contributions of parameters measured in different units over a probabilistic space. The conditional probability under consideration can be used for testing the given dependency: if it is greater than a certain threshold, then we accept the claim that the outcome of measurements correspond to person P. However, we are also interested in testing the independence of the input and the output parameters. In this case, the algorithm, which is based on the computation of the product

$$
\operatorname{Pr}\{(\hat{\mathrm{H}}, \hat{\mathrm{W}})=(\hat{\mathrm{h}}, \hat{\mathrm{w}})\}=\operatorname{Pr}_{\mathrm{h}}\{\hat{\mathrm{h}}\} \underset{\mathrm{w}}{\operatorname{Pr}\{\hat{\mathrm{w}}\}}
$$


and comparison it with the threshold fails (for example, the marginal probability distributions of parameters can be uniform).

2. Suppose that there are 3 hypotheses about person P. Namely, we assume that

$$
\mathrm{P} \in\{\mathrm{A}, \mathrm{B}, \text { neither } \mathrm{A} \text { nor } \mathrm{B}\} .
$$

Having received the observations, an algorithm has to check whether they belong to the decision regions of $\mathrm{A}$ or $\mathrm{B}$. Thus, these decision regions have to be disjoint, i.e., the decision about the person $\mathrm{A}$ cannot be made without taking into account the person $\mathrm{B}$ and vise versa. Furthermore, the conditional probabilities for "neither A nor B" are not defined. In the biometric authentication problem we have only parameters of a fixed person involved. Nevertheless, since we also want to evaluate the false acceptance rate, the scheme above is relevant: "person $\mathrm{A}$ " is the joint probability distribution $f_{\mathbf{x}}(\mathbf{x}) f_{\mathbf{y}}(\mathbf{y})$, "person $\mathrm{B}$ " is the joint probability distribution $f_{\mathbf{x}}(\mathbf{x}) f_{\mathbf{y} \mid \mathbf{x}}(\mathbf{y} \mid \mathbf{x})$. The space where the decision regions are constructed is the probabilistic space.

3. The basic step of our approach to solving the authentication problem uses the constructions of pseudo-random generators. Let $F^{*}$ be an arbitrary function satisfying the constraints: (a) $F^{*}$ is defined over the interval $I$ where a random variable $X$ can take values; (b) $F^{*}(x) \in[0,1]$ for all $x \in I$; (c) $F^{*}$ is a strictly increasing function over $I$. Thus, $F^{*}$ is a PD of some random variable $X^{*}$ and if $z \in(0,1)$, then the equation $z=F^{*}(x)$ has a unique solution for $x \in I$. Furthermore, if $F^{*}=F$, where $F$ is the $\mathrm{PD}$ of the random variable $X$, then $F(X)$ is a random variable uniformly distributed over the $[0,1]$ interval. As a result, if our hypotheses about probability distributions of components of the vectors $\mathbf{x}$ and $\mathbf{y}$ are correct, then one of the distortion functions, $\Delta_{\mathrm{i}}$ or $\Delta_{\mathrm{d}}$, is distributed according to the vector $\boldsymbol{\beta}$, which is completely determined by the parameter $q$.

4. The feature of biometric authentication is making the decisions by investigation of samples of small sizes. Our technique allows us to carry out such an analysis, since all probabilities under consideration are expressed exactly using the generating functions of discrete random variables. An introduction of the parameter $q$ in the $\xi$ transformation also brings data compression.

5. Notice that optimization over possible assignments of the distortion functions $\Delta_{\mathrm{i}}$ and $\Delta_{\mathrm{d}}$ introduced in (13), (14) is possible and it results in the improvement of the performance, when (9) is understood as a criterion. In particular, if the absolute values of the differences at the right-hand sides of (13), (14) are taken to the power $\alpha$, then the optimum assignment is $\alpha \approx 1.7$ for the example given in the previous section.

\section{References}

1. Bolle, R.M., Connell, J.H., Pankanti, S., Ratha, N.K., Senior, A.W.: Guide to Biometrics. Springer, New York (2004)

2. Kullback, S.: Information Theory and Statistics. Dover, New York (1968) 